\title{
Impacto do programa de fisioterapia aquática funcional em idosos com hipertensão arterial
}

http://dx.doi.org/10.11606/1807-5509201800040513

\author{
Bianca Ferdin CARNAVALE* \\ Bruna PIANNA* \\ Camila GIMENES* \\ Silvia Regina BARRILE* \\ Guilherme Eleutério ALCALDE* \\ Júlia Moretto MORATELLI* \\ Eduardo Aguilar ARCA*
}

\section{Resumo}

0 objetivo do estudo foi verificar a influência do programa de fisioterapia aquática na aptidão funcional, pressão arterial e qualidade de vida em idosos hipertensos. Os voluntários foram alocados por conveniência em dois grupos: grupo aquático (GA), que foram submetidos ao programa de fisioterapia aquática e o grupo controle (GC). A coleta de dados constituiu das medidas da pressão arterial, aplicação dos testes de caminhada de 6 minutos (TC6), flexibilidade, timed up and go (TUG) e questionário de qualidade de vida WHOQOL-bref, nos momentos pré e pós-intervenção aquática. 0 programa de intervenção teve duração de 16 semanas, sendo constituído por exercícios aeróbicos e funcionais. Para os dados paramétricos, foram utilizados os testes $t$ de Student para duas amostras independentes para comparação dos grupos e duas amostras pareadas para comparação dos momentos. Para os dados não paramétricos foram aplicados os testes de Mann-Whitney para comparação dos grupos e Wilcoxon para comparação dos momentos. Em todos os testes, foi considerado resultado estatisticamente significante quando $p<0,05$. Na comparação entre os grupos houve diferença nos momentos pós do teste de flexibilidade de $18,95(10,20-36,03) \mathrm{cm}$ no GA para $10,50(5,73-21,83) \mathrm{cm}(\mathrm{p}=0,02)$ no GC. Na comparação entre os momentos houve modificações dos valores do teste de flexibilidade de 11,80 $(3,0-27,30) \mathrm{cm}$ para $15,05(10,20-36,03) \mathrm{cm}(\mathrm{p}=0,01)$ e do TC6 de 409,25 $(300,0-600,0) \mathrm{m}$ para 488,50 $(349,0-616,0) m(p=0,002)$ no GA. Pode-se inferir que a fisioterapia aquática contribui para a melhora da aptidão funcional em idosos com doenças crônicas não transmissiveis.

Palavras-Chave: Hidroterapia; Pressão Arterial; Envelhecimento; Amplitude de Movimento Articular; Qualidade de Vida.

\section{Introdução}

Atualmente observa-se aumento da prevalência e incidência de Doenças Crônicas Não Transmissíveis (DCNT) no mundo, devido ao envelhecimento populacional e as consequentes modificações nos padrốes de morbidade, invalidez e mortalidade ${ }^{1}$.

Dentre as DCNT destaca-se a hipertensão arterial (HA) que acomete aproximadamente 30\% da população mundial adulta, sendo que essa prevalência aumenta a partir dos 60 anos de idade ${ }^{2}$.

A HA está relacionada com o declínio da capacidade funcional (CF) de indivíduos idosos, sendo necessária a avaliação e acompanhamento das atividades de vida diária (AVD) ${ }^{3}$. Os indivíduos com hipertensão apresentam piora na qualidade de vida $(\mathrm{QV})$ relacionada à saúde quando comparados com indivíduos com pressão arterial normal, podendo essa condiçáo ser agravada quando a HA está associada com outras comorbidades ${ }^{4}$.

Assim sendo, a fisioterapia aquática é indicada para idosos com hipertensão, pelo fato de apresentar inúmeros benefícios a saúde funcional, como aumento da força muscular, flexibilidade, controle da pressão arterial e melhora da qualidade de vida ${ }^{5-8}$.

Os exercícios aquáticos podem ser considerados como a forma mais segura de treinamento muscular, pois promovem resistência multidimensional constante durante a realizaçáo dos movimentos com uma sobrecarga mínima nas articulaçóes ${ }^{8}$. 
Contudo, pouco se sabe sobre os efeitos do programa de fisioterapia aquática, que utiliza cicloergômetros aquáticos e exercícios funcionais em idosos com hipertensão arterial.

\section{Método}

\section{Aspectos éticos}

Antes de iniciar a coleta de dados, todos os voluntários assinaram o Termo de Consentimento Livre e Esclarecido. O estudo foi aprovado pelo Comitê de Ética em Pesquisa da Universidade do Sagrado Coração (USC), por meio da Plataforma Brasil, CAAE: 30282214.3 .0000 .5502 (parecer n ${ }^{\circ}$ 616.307).

\section{Delineamento}

Trata-se de um estudo quase experimental, do tipo antes e depois, com amostragem escolhida por conveniência.

Foram voluntários do estudo, idosos que apresentavam hipertensão arterial, encaminhados ao serviço de fisioterapia da Universidade do Sagrado Coração (USC) da cidade de Bauru, SP, Brasil. Após a seleção, os mesmos foram alocados por conveniência em dois grupos: Grupo Aquático (GA): os participantes foram submetidos ao programa de fisioterapia aquática e Grupo Controle (GC): os participantes não realizaram nenhum tipo de intervenção (inativo).

Foram selecionados 21 voluntários idosos com hipertensão arterial, 11 foram alocados no GA e 10 no GC. Houve uma perda no GA e duas no GC por motivo de desistência. A coleta de dados foi finalizada com 18 voluntários, 10 (3 homens e 7 mulheres) no GA e oito ( 2 homens e 8 mulheres) no GC.

A média de idade dos voluntários do GA foi de $66,4 \pm 7,1$ anos e do GC foi de 67,8 \pm 7,1 anos. De acordo com o IMC, a maioria foi classificada como obesos (70\% do GA e 75\% do GC). Todos os voluntários usavam medicamentos anti-hipertensivos e relataram outras doenças como a diabetes mellitus tipo $2(72 \%)$, lombalgia (22\%), osteoartrite de joelho $(38 \%)$ e osteoporose (11\%).

Não foi possível realizar a aleatorização da amostra, pois alguns voluntários declararam na avaliação inicial, que não tinham disponibilidade de tempo para participar do grupo de intervenção, desde modo, os mesmos foram alocados por conveniência no grupo controle.
Dessa forma, o objetivo do presente estudo foi verificar a influência do programa de fisioterapia aquática na aptidão funcional, pressão arterial e qualidade de vida em idosos hipertensos.

Por questôes éticas, após o término do período intervenção foi oferecido para os voluntários do GC, sem ônus financeiro, a participação de um programa com os mesmos procedimentos realizados no GA.

\section{Cálculo do tamanho da amostra}

O cálculo foi estimado considerando-se a possibilidade de melhora em $20 \%$ na flexibilidade a favor do grupo de intervençáo em relação ao grupo controle. Para isso, o erro tipo I (alfa) foi estimado em $5 \%$ e o poder do teste de $80 \%$. O número total de participantes estimado para cada grupo foi de 10 .

\section{Critérios de inclusão e exclusáo}

Foram incluídos idosos com diagnóstico médico de hipertensão arterial, nos estágios I e II, segundo os critérios de classificação das VI Diretrizes Brasileiras de Hipertensão ${ }^{2}$ e que não apresentaram nenhum comprometimento funcional que impedisse a execução do protocolo de intervenção. Além disso, os voluntários não realizaram nenhum tipo de dieta alimentar restrita, assim como, não participaram de outros programas de exercícios físicos regulares.

Foram excluídos aqueles que apresentaram as seguintes contraindicaçôes para o programa de exercícios aquáticos: otite, hidrofobia, feridas cutâneas, micoses e hipertensão estágio III $^{2}$. Também foram excluídos os voluntários que por qualquer motivo, mudaram as doses ou a classe dos anti-hipertensivos empregados, tendo em vista que tais alteraçóes podem influenciar intensamente as variáveis pressóricas estudadas, assim como, aqueles que tiveram mais do que seis faltas do programa de exercícios.

\section{Procedimento da coleta de dados}

A pressão arterial (PA) foi medida por meio de um aparelho de pressão (Lane Instruments ${ }^{\circledR}$ ) e estetoscópio (Littmann Classic III'), seguindo a recomendação das VI Diretrizes Brasileiras de Hipertensão ${ }^{2}$. 
A flexibilidade foi avaliada pelo teste de Wells e Dillon ${ }^{9}$, também denominado teste de sentar e alcançar (Sit-and-reach Test). Para a realização do teste, os participantes sentaram no chão com os joelhos estendidos, membros inferiores levemente afastados com os pés apoiados na parede da caixa de madeira (banco de Wells - Sanny'), cotovelos estendidos e ombros fletidos. Em seguida foi realizada a flexão de tronco, avançando lentamente para frente com ambas as mãos (paralelas), tão longe quanto possível, mantendo essa posição momentaneamente. Foi registrada a média das três execuçóes do teste de cada indivíduo.

Para avaliar a resistência aeróbica e capacidade funcional foi utilizado o teste de caminhada, que avalia a distância máxima percorrida em 6 minutos andando. Os equipamentos utilizados para realizar o teste foram: cronômetro (Nautika Procon ${ }^{\circledR}$ ), cones para delimitaçáo do circuito, esfigmomanômetro (Lane Instruments'), estetoscópio (Littmann Classic III') e oxímetro portátil (PM100C Visor Oled colorido $\mathrm{New}$ Tech). Antes da realização do teste, os pacientes fizeram um período de repouso de 10 minutos, neste tempo foram avaliados os dados de pressáo arterial, oximetria de pulso, nível de dispneia, segundo a Escala de Borg ${ }^{10}$, frequência cardíaca e respiratória. Durante o teste, a cada dois minutos foram avaliados a frequência cardíaca, saturação de oxigênio e nível de dispneia. Ao término, os dados vitais coletados inicialmente foram novamente avaliados, em seguida, foi calculada a distância total caminhada pelo voluntário ${ }^{11}$.

Também para avaliar a capacidade funcional foi utilizado o teste Timed Up and Go (TUG), quantifica o tempo gasto no percurso de 3 metros. O voluntário inicia o teste em posiçáo sentada em postura ereta, ao sinal indicado o paciente levanta da cadeira, caminha até um marcador, contorna-o, retorna à cadeira e senta-se, encosta as costas na cadeira novamente ${ }^{12}$.

Para a avaliação da qualidade de vida foi utilizado o questionário WHOQOL-bref, que é composto por 26 perguntas referentes aos quatro domínios: físico, psicológico, social e ambiental ${ }^{13}$.

Para calcular a frequência cardíaca máxima (FCmáx) na água foi utilizado o seguinte modelo matemática, proposto por GraEf e KRUEL ${ }^{14}$ : FCmáx na água $=$ FCmáx em terra $-\triangle \mathrm{FC}$ (bradicardia decorrente da imersão). Para a realização da medida da FC, foi utilizado um monitor cardíaco (Polar FT $1^{\circledR}$ ). Os voluntários permaneceram imersos na posição ortostática, em repouso, com água no nível do processo xifoide. Durante a avaliação e atendimentos, a temperatura da água foi controlada por um termostato, sendo mantida em $32,5^{\circ} \mathrm{C}$.
As informaçóes para a caracterização da amostra foram coletadas por meio de uma entrevista. $\mathrm{O}$ mesmo pesquisador foi o responsável por realizar todos os testes, medidas avaliativas e aplicar o questionário de qualidade de vida, descritos anteriormente. A reavaliação (pós-intervençáo) seguiu os mesmos procedimentos da avaliação inicial (pré-intervenção).

\section{Programa de fisioterapia aquática (PFA)}

O PFA foi baseado nos estudos realizados por Adsett et $\mathrm{al}^{15}$, Cugusi et $\mathrm{al}^{16}$ e Sattar, Esfarjani e Nezakatalhosseini ${ }^{17}$. O período de intervenção foi de 16 semanas, periodicidade de três vezes por semana (dias alternados). O PFA foi constituído de duas etapas distintas:

Etapa 1 - Condicionamento cardiovascular: com duraçáo de 30 minutos. Os sujeitos realizaram exercícios nas bicicletas aquáticas (Hidrobike ${ }^{\circledR}$ ). A intensidade dos exercícios permaneceu entre 60\% a $80 \%$ da $\mathrm{FC}_{\text {máx }}$ na água, sendo monitorada por meio da palpaçáo da artéria radial a cada 10 minutos.

Etapa 2 - Exercícios funcionais: esta etapa teve a duração de aproximadamente 30 minutos e foi dividida em seis componentes: 1. Flexibilidade: foram realizados alongamentos dos grupos musculares: tríceps braquial, peitoral maior, quadríceps, isquiotibiais, gastrocnêmio e adutores da coxa. Foram realizadas seis séries de 10 segundos de manutenção (alongamento estático) para cada grupo muscular e 10 segundos de descanso (entre as séries). 2. Coordenaçáo motora e agilidade: os participantes fizeram deslocamentos no sentido diagonal, anteroposterior e exercícios de agachamento na parte rasa da piscina (1 metro de profundidade). Em seguida, subiram e desceram a escada interna da piscina, alternando pernas (2 degraus). Foram realizadas quatro séries de 30 segundos ativos (para cada exercício) e 30 segundos de descanso (entre as séries). 3. Percepçáo espaço - temporal e velocidade de reaçáo: os voluntários foram subdivididos em duplas para arremessar uma bola. Os arremessos foram realizados associados com a execuçáo da marcha com deslocamentos ântero-posteriores e látero-laterais na parte rasa da piscina. Foram realizadas três séries de um minuto ativo e 30 segundos de descanso (entre as séries). 4. Equilíbrio: Os voluntários permaneceram em posição ortostática com os pés em cima de pranchas $\left(\right.$ Floty $^{\circledR}$ ) para treino de equilíbrio. $\mathrm{O}$ apoio foi bipodal e unipodal. Foram realizadas quatro séries de um minuto ativo e 30 segundos de descanso (entre as séries). 5. Fortalecimento muscular: 
a) Tríceps braquial: foram utilizados aquatubos $\left(\right.$ Floty ${ }^{\circledR}$ ) para a execução dos exercícios. Os voluntários permaneceram em pé na parte intermediária da piscina $(1,40 \mathrm{~cm})$ com os ombros aduzidos e com cotovelos próximos ao tronco, realizaram movimentos de extensão e flexão, mantendo os antebraços pronados. b) Peitoral Maior: na posição ortostática, com flexão de ombro a $90^{\circ}$, segurando a prancha de E.V.A. (Floty ${ }^{\oplus}$ ), foram realizados movimentos de flexão e extensão de cotovelo (aproximando e afastando a prancha do tronco). c) Quadríceps e glúteos: na mesma posição descrita anteriormente, segurando na barra fixa na parte rasa da piscina, foi utilizado aquatubos (flutuadores) na região plantar, sendo realizados movimentos de extensão e flexão de joelho (alternando os membros). d) Adutores da coxa: posição idêntica ao exercício anterior, apoiados na barra fixa, foi utilizada tornozeleiras de E.V.A. (Floty ${ }^{\circledR}$ ) nos membros inferiores, sendo realizados movimentos de abdução e adução, com o joelho em extensão e os pés em dorsiflexão (alternando os membros). e) Reto abdominal: Para a realização dos exercícios abdominais, os voluntários apoiaram as mãos na barra fixa da piscina e realizaram movimentos de "chutes", tocando bilateralmente os pés na parede da piscina e em seguida retornando ao chão. Foram realizadas seis séries de 10 segundos ativos e 10 segundos de descanso (entre as séries). 6. Relaxamento: os voluntários permaneceram em repouso por 5 minutos, sendo utilizado turbilhão para hidromassagem.

\section{Análise estatística}

Os resultados deste estudo foram analisados pelo programa Statistical Package for Social Sciences para Windows, versão 18.0 (SPSS Inc, Chicago, IL). A normalidade dos dados foi verificada com o teste Shapiro-Wilk. Os dados paramétricos foram expressos em média e desvio padrão, sendo utilizado o teste $t$ de Student para duas amostras independentes para comparação dos grupos e duas amostras pareadas para comparação dos momentos. Os dados não paramétricos foram expressos em mediana, valores máximos e mínimos. Para comparaçáo dos grupos foi utilizado o teste de Mann-Whitney e para comparação dos momentos foi aplicado o teste de Wilcoxon. Em todos os testes, foi considerado resultado estatisticamente significante quando $\mathrm{p}<0,05$.

\section{Resultados}

Na TABELA 1 pode ser observado que não houve diferença estatisticamente significante na pressão arterial em todas as comparaçôes.

Na TABELA 2 pode ser constatada diferença estatisticamente significante do teste de flexibilidade e do TC6 no GA.
Na TABELA 3 pode ser observado que não houve diferença estatisticamente significante nos domínios de qualidade de vida em todas as comparaçóes.
GA: Grupo Aquático; GC: Grupo Controle; PAS: Pressão Arterial Sistólica;

PAD: Pressão Arterial Diastólica. Os dados são apresentados em média e desvio padrão $p>0,05$ em todas as comparações.

GA: Grupo Aquático; GC: Grupo Controle; TUG: Timed UP and Go; TC6: Teste de Caminhada de 6 Minutos. Os dados são apresentados em mediana (mínimomáximo). As letras
TABELA 1 - Medidas da pressão arterial

\begin{tabular}{lcccc}
\hline \multirow{2}{*}{ Variáveis } & \multicolumn{2}{c}{ GA $(\mathbf{n}=\mathbf{1 0})$} & \multicolumn{2}{c}{ GC $(\mathbf{n}=\mathbf{8})$} \\
\cline { 2 - 5 } & Pré & Pós & Pré & Pós \\
\hline PAS $(\mathrm{mmHg})$ & $134,0 \pm 9,6$ & $128,4 \pm 10,7$ & $136,7 \pm 16,3$ & $139,2 \pm 15,2$ \\
PAD $(\mathrm{mmHg})$ & $80,0 \pm 9,4$ & $74,4 \pm 7,6$ & $80,2 \pm 4,9$ & $77,2 \pm 7,1$ \\
\hline
\end{tabular}

TABELA 2 - Medidas das variáveis de aptidão funcional

\begin{tabular}{lcccc}
\hline \multirow{2}{*}{ Variáveis } & \multicolumn{2}{c}{ GA $(\mathbf{n}=\mathbf{1 0})$} & \multicolumn{2}{c}{ GC $(\mathbf{n}=\mathbf{8})$} \\
\cline { 2 - 5 } & Pré & Pós & Pré & Pós \\
\hline Flexibilidade $(\mathrm{cm})$ & $12,30 \mathrm{a}(3,0-27,30)$ & $18,95 \mathrm{Aa}(10,20-36,03)$ & $10,15(6,23-23,0)$ & $10,50 \mathrm{~A}(5,73-21,83)$ \\
\multirow{2}{*}{ TUG $(\mathrm{s})$} & 7,90 & 7,50 & 10,10 & 9,80 \\
& $(5,28-12,33)$ & $(5,40-11,37)$ & $(8,90-13,88)$ & $(7,47-13,41)$ \\
\hline
\end{tabular}


TABELA 2 - Medidas das variáveis de aptidão funcional

\begin{tabular}{lcccc}
\hline \multirow{2}{*}{ Variáveis } & \multicolumn{2}{c}{ GA $(\mathbf{n}=\mathbf{1 0})$} & \multicolumn{2}{c}{ GC $(\mathbf{n}=\mathbf{8})$} \\
\cline { 2 - 5 } & Pré & Pós & Pré & Pós \\
\hline \multirow{2}{*}{ TC6 $(\mathrm{m})$} & $409,25 \mathrm{~b}$ & $488,50 \mathrm{~b}$ & 437,0 & 459,0 \\
& $(300,0-600,0)$ & $(349,0-616,0)$ & $(246,0-553,0)$ & $(368,0-588,0)$ \\
\hline
\end{tabular}

TABELA 3 - Domínios de qualidade de vida

\begin{tabular}{lcccc}
\hline \multirow{2}{*}{ Domínios } & \multicolumn{2}{c}{ GA $(\mathbf{n}=\mathbf{1 0})$} & \multicolumn{2}{c}{ GC $(\mathbf{n}=\mathbf{8})$} \\
\cline { 2 - 5 } & Pré & Pós & Pré & Pós \\
\hline \multirow{2}{*}{ Físico } & 66,1 & 60,7 & 57,1 & 64,3 \\
& $(53,6-82,1)$ & $(46,4-75,0)$ & $(42,0-75,0)$ & $(39,3-71,4)$ \\
Psicológico & 66,7 & 66,7 & 66,7 & 62,5 \\
& $(41,7-87,5)$ & $(41,7-79,2)$ & $(45,8-79,2)$ & $(58,3-66,7)$ \\
Social & 75,0 & 75,0 & 79,1 & 75,0 \\
& $(33,3-83,3)$ & $(66,7-91,7)$ & $(50,0-100,0)$ & $(66,7-100,0)$ \\
Ambiental & 68,8 & 71,9 & 68,8 & 68,8 \\
& $(50,0-100,0)$ & $(53,1-84,4)$ & $(50,0-84,4)$ & $(56,3-87,5)$ \\
\hline
\end{tabular}

\section{Discussão}

O propósito do estudo foi verificar a influência do programa de fisioterapia aquática na aptidão funcional, pressão arterial e qualidade de vida em idosos hipertensos. Foi constatado que o programa de intervenção promoveu resultados satisfatórios na flexibilidade e resistência aeróbica.

O resultado referente ao aumento da flexibilidade obtida pelos voluntários do GA pode ser atribuído às propriedades físicas da água, principalmente o empuxo, que é o responsável pela redução da sobrecarga articular e a temperatura elevada da água que favorece a diminuição do espasmo doloroso, diminuindo o tônus muscular e facilitando a amplitude de movimento articular ${ }^{6,8,18}$.

Além disso, os exercícios de alongamentos realizados no programa de intervenção aquático foram estratégias importantes para o aumento da flexibilidade na musculatura da cadeia posterior de tronco e membros inferiores.

Com relaçáo à resistência aeróbica, avaliada pelo TC6, houve aumento na distância percorrida pelos voluntários do GA após o programa de intervenção. Este resultado pode ser explicado devido ao fato que cada sessão, foi realizada 30 minutos de exercícios em circloergômetros aquáticos. Esses dados confirmam a eficácia da fisioterapia aquática na melhora da capacidade aeróbica e funcional nessa população ${ }^{19}$.
Com relação ao TUG, não houve diferença estatisticamente significante em ambos os grupos, provavelmente pelo fato dos voluntários apresentarem parâmetros de normalidade ${ }^{12}$ na pré-intervenção, ou seja, os valores obtidos na avaliação inicial foram inferiores a 10 segundos.

Nossos achados divergem da pesquisa de Alcalde e Guimaráes ${ }^{20}$ que aplicaram o TUG em idosos hipertensos, obtendo diminuiçấo significativa no tempo de execução do teste no grupo de intervenção aquática. O programa consistiu essencialmente de exercícios de agilidade, coordenação e equilíbrio dinâmico, sendo que tais elementos foram fundamentais para o bom desempenho obtido pelo TUG.

Também não houve diferença estatisticamente significante nas variáveis pressóricas, porém, de acordo com as Diretrizes Brasileiras de Hipertensão ${ }^{2}$ houve decréscimo nos valores, com reclassificação da PAS do GA de limítrofe para normal e PAD de normal para ótima. Segundo Whelton et al..$^{21} \mathrm{e}$ Corlinessen e SMART ${ }^{22}$, em revisóes sistemáticas, a redução da pressão arterial após exercício físico é em média de 3,84 (PAS)/2,58(PAD) $\mathrm{mmHg}$ e 3,5(PAS)/2,5(PAD) $\mathrm{mmHg}$, respectivamente. Comparando com as medidas da PA do presente estudo, observamos reduçóes de $6 \mathrm{mmHg}$ da PAS e PAD do GA. minúsculas iguais indicam diferença estatisticamente significante: a $(p=0,01) ; b$ $(p=0,002)$ na comparação entre os momentos em relação aos grupos (Teste de Wilcoxon). As letras maiúsculas iguais indicam diferença estatisticamente significante: $A$ ( $p=0,02)$ na comparação entre os grupos em relação aos momentos (Teste de Mann Whitney).

GA: Grupo Aquático; GC: Grupo Controle. Os dados são apresentados em mediana (mínimomáximo). $p>0,05$ em todas as comparações. 
Os resultados satisfatórios da flexibilidade e resistência aeróbica, não foram suficientes para que houvesse a percepção de melhora na qualidade de vida relacionada a saúde dos voluntários do GA. Contudo, sabe-se que a fisioterapia aquática é indicada para a manutenção ou melhora da qualidade de vida em idosos, especialmente no domínio psicológico, pois geralmente os programas de exercícios são realizados em grupo, fato que promove a integração social ${ }^{23}$.

Como limitação e deficiências do estudo, pode ser apontado o método de controle da intensidade do treinamento aeróbico, visto que os cicloergômetros aquáticos não tinham dispositivos para o monitoramento da frequência cardíaca. Porém, sistematicamente, a cada 5 minutos durantes as sessóes, a própria pesquisadora realizou a palpação da artéria radial, para que os voluntários se mantivessem na zona de alvo de treinamento preestabelecido.

Por outro lado, o estudo apresentou alguns pontos fortes, como a descrição detalhada do protocolo de exercícios, fato que permite sua reprodutibilidade e aplicabilidade na prática clínica, pois é indicado para essa população, pelo fato possuir componentes que visam o condicionamento cardiorrespiratório, aumento da flexibilidade, melhora do equilíbrio estático e dinâmico, coordenação e agilidade e resistência muscular.

Dessa forma, o exercício realizado no ambiente aquático, é um valioso recurso terapêutico que produz benefícios à saúde funcional de idosos com doenças crônicas não transmissíveis.

\section{Abstract \\ Impact of functional aquatic therapy program in older people hypertensive patients}

The objective of the study was to investigate the influence of the aquatic therapy program in functional fitness, blood pressure and quality of life in older people hypertensive patients. The volunteers were divided for convenience into two groups: water group (WG), which were submitted to aquatic physical therapy program and the control group (CG). The intervention program was 16 weeks long, consisting of aerobic and functional exercises. For parametric data, Student t test for independent samples to compare groups and paired samples to compare the times were used. For nonparametric data were applied the Mann-Whitney test to compare the groups and Wilcoxon for comparison of moments. In all tests, it was considered statistically significant result when $p<0.05$. In the comparison between the groups was no difference in the post time of the flexibility test $18.95(10.20-36.03)$ in WG to $10.50 \mathrm{~cm}(5.73-21.83) \mathrm{cm}(p=0.02)$ in CG. Comparing the times there were changes in the values of flexibility test of $11.80(3.0-27.30) \mathrm{cm}$ to $15.05(10.20-36.03) \mathrm{cm}$ $(p=0.01)$ and 6 WT of $409.25(300.0-600.0) \mathrm{m}$ to $488.50(349.0-616.0) \mathrm{m}(\mathrm{p}=0.002)$ in WG. Can be inferred that the aquatic physical therapy contributes to the improvement of functional capacity in older people with chronic non-communicable diseases.

KEYwORDS: Hydrotherapy; Arterial Pressure; Aging; Joint Range of Motion; Quality of Life.

\section{Agradecimentos}

A Hidrobike Comércio de Equipamentos Esportivos Ltda pela cooperação e parceria. Ao Programa Institucional Voluntário de Iniciação Científica da Universidade do Sagrado Coração (PIVIC/USC) pelo apoio e viabilidade do projeto de pesquisa.

\section{Referências}

1. Rodriguaes MAP, Facchini LA, Piccini RX, et al. Uso de serviços básicos de saúde por idosos portadores de condiçôes crônicas, Brasil. Rev Saúde Pública. 2009;43(4):604-12. 
2. Sociedade Brasileira de Cardiologia; Sociedade Brasileira de Hipertensão; Sociedade Brasileira de Nefrologia. VI Diretrizes Brasileiras de Hipertensão. Arq Bras Cardiol. 2010; 95 (1 supl 1):1-51.

3. Pedrosa R, Holanda G. Correlação entre os testes de caminhada, marcha estacionária e TUG em hipertensas idosas. Rev Bras Fisioter. 2009;13(3):252-6.

4. Carvalho MV, Siqueira LB, Sousa ALL, Jardim PCBV. A influência da hipertensão arterial na qualidade de vida. Arq Bras Cardiol. 2013;100(2):164-74.

5. Arca EA, Martinelli B, Martin LC, Waisberg CB, Franco RJ. Aquatic Exercise is as Effective as dry Land Training to Blood Pressure Reduction in Postmenopausal Hypertensive Women. Physiother Res Int. 2014;19(2):93-8.

6. Arca EA, Fiorelli A, De Vitta A, et al. Efetividade do Programa de Fisioterapia Aquática na amplitude de movimento em idosas. Kairós. 2013;16(5):73-82.

7. Bocalini DS, Serra AJ, Rica LR, Santos L. Repercussions of training and detraining by water-based exercise on functional fitness and quality of life: a short-term follow-up in healthy older women. Clinics. 2010;65(12):1305-09.

8. Candeloro JM, Caromano FA. Efeito de um programa de Hidroterapia na flexibilidade e força muscular de idosas. Rev Bras Fisioter. 2007;11(4):303-9.

9. Wells KF, Dillon KE. The sit and reach: a test of back and leg flexibility. Res Q Exerc Sport. 1952;23(1):115-8.

10. Borg GAV. Psychophysical bases of perceived exertion. Med Sci Sports Exerc. 1982;14(5):377-81.

11. American Thoracic Society. ATS Statement: Guidelines for the Six-Minute Walk Test. Am J Respir Crit Care Med. 2002;166(1):111-7.

12. Bohannon RW. Reference values for the Timed Up and Go test: a descriptive meta-analysis. J Geriatr Phys Ther. 2006;29(2):64-8.

13. 13. Fleck MPA, Louzada S, Xavier M, et al. Aplicação da versão em Português do instrumento abreviado de avaliação de qualidade de vida "WHOQOL-bref”. Rev Saúde Pública. 2000;34(2):178-83.

14. Graef FI, Kruel LFM. Frequência cardíaca e percepção subjetiva do esforço no meio aquático: diferenças em relação ao meio terrestre e aplicações na prescrição do exercício - uma revisão. Rev Bras Med Esporte. 2006;12(4):221-8.

15. Adsett JA, Mudge AM, Morris N, Kuys S, Paratz JD. Aquatic exercise training and stable heart failure: A systematic review and meta-analysis. Int J Cardiol. 2015;186:22-8.

16. Cugusi L, Cadeddu C, Nocco $S$, et al. Effects of an aquatic-based exercise program to improve cardiometabolic profile, quality of life, and physical activity levels in men with type 2 diabetes mellitus. PM R. 2015;7(2):141-8.

17. Sattar M, Esfarjani F, Nezakatalhosseini M. The effect of aquatic-resistance training on quality of life in postmenopausal women. Procedia Soc Behav Sci. 2013;70:1732-9.

18. Becker BE. Aquatic Therapy: Scientific Foundations and Clinical Rehabilitation Applications. PM R. 2009;1(9):859-72.

19. Malta P, Silva RCP. Avaliação da capacidade aeróbica em idosas submetidas à hidrocinesioterapia [trabalho de conclusão de curso]. Belém (PA): Universidade da Amazônia; 2009.

20. Alcalde GE, Guimarães LE. Avaliação da capacidade funcional de idosos submetidos a um programa de fisioterapia aquática [trabalho de conclusão de curso]. Bauru (SP): Universidade do Sagrado Coração; 2014.

21. Whelton SP, Chin A, Xin X, He J. Effect of aerobic exercise on blood pressure: a meta-analysis of randomized, controlled trials. Ann Intern Med. 2002;136(7):493-503.

22. Cornelissen VA, Smart NA. Exercise training for blood pressure: a systematic review and meta-analysis. J Am Heart Assoc. 2013 Feb 1;2(1):e004473. doi: 10.1161/JAHA.112.004473.

23. Fibra T, SáTSTF, Fontes SV, Driusso P, Prado GF. Avaliação da qualidade de vida de idosos submetidos à fisioterapia aquática. Rev Neurocienc. 2006;14(4):182-4.

\begin{tabular}{r|r} 
ENDEREço & \\
Bianca Ferdin Carnavale & Recebido para publicação: 14/04/2016 \\
Universidade do Sagrado Coração & Revisado: 30/06/2016 \\
Rua Irmã Arminda, 10-50- Jardim Brasil & Aceito: 08/o8/2016 \\
17011-160 - Bauru - São Paulo - BRASIL & \\
e-mail: bianca.ferdin@hotmail.com & \\
\hline
\end{tabular}

